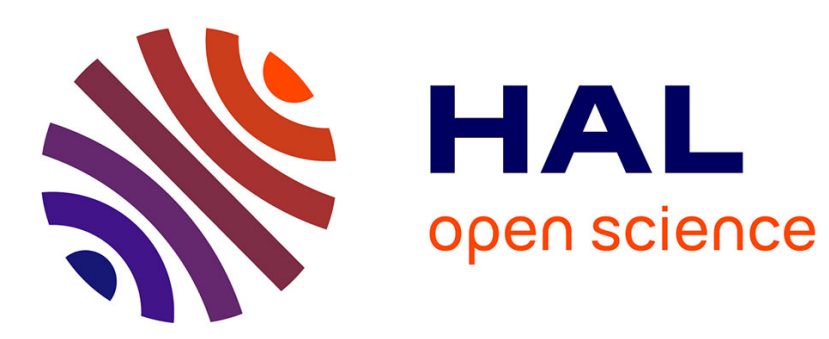

\title{
Autour du Fojiao chuchuan nanfang zhi lu wenwu tulu
} Li-Ying Kuo

\section{To cite this version:}

Li-Ying Kuo. Autour du Fojiao chuchuan nanfang zhi lu wenwu tulu. Arts Asiatiques, 1998, 53 (1), pp.102 - 111. 10.3406/arasi.1998.1425 . halshs-02513080

\section{HAL Id: halshs-02513080 \\ https://shs.hal.science/halshs-02513080}

Submitted on 20 Mar 2020

HAL is a multi-disciplinary open access archive for the deposit and dissemination of scientific research documents, whether they are published or not. The documents may come from teaching and research institutions in France or abroad, or from public or private research centers.
L'archive ouverte pluridisciplinaire HAL, est destinée au dépôt et à la diffusion de documents scientifiques de niveau recherche, publiés ou non, émanant des établissements d'enseignement et de recherche français ou étrangers, des laboratoires publics ou privés. 


\section{Autour du Fojiao chuchuan nanfang zhi lu wenwu tulu}

Li-ying Kuo

\section{Citer ce document / Cite this document :}

Kuo Li-ying. Autour du Fojiao chuchuan nanfang zhi lu wenwu tulu. In: Arts asiatiques, tome 53, 1998. pp. 102-111; doi : https://doi.org/10.3406/arasi.1998.1425

https://www.persee.fr/doc/arasi_0004-3958_1998_num_53_1_1425

Fichier pdf généré le 21/04/2018 


\section{Autour du Fojiao chuchuan nanfang zhi lu wenwu tulu ${ }^{(1)}$}

(«Catalogue d'objets [décorés] d’images [venues] par la voie du sud au début de la transmission du bouddhisme [en Chine|»). Beijing : Wenwu chubanshe, 1993, 191 p. (127 pl.), 2 préfaces. ISBN 7-5010-0718-7/K. 304.

Les revues Wenw'u («Patrimoine culturel») et Kaogu («Archéologie») sont indispensables pour la connaissance des découvertes archéologiques faites en Chine. I.es rapports que ces revues publient sont parfois sommaires et les photographies sont trop souvent médiocres, au point d'être inutilisables. C"est pourquoi il faut se réjouir de la publication de ce catalogue d'images bouddhiques trouvées en Chine du Sud et datant des premiers temps de la transmission du bouddhisme en Chine. Treize ans se sont écoulés depuis que M. Wu Hong a attiré l'attention des historiens d'art sur l'existence d'éléments bouddhiques dans l'art chinois des II $^{\mathrm{e}}$ et $\mathrm{III}^{\mathrm{e}}$ siècles'. Depuis, d'autres découvertes archéologiques ont été publiées ainsi que quelques études spécialisées. Je les examinerai dans la présente chronique, en particulier la plus importante d'entre elles, ce Catalogue d'objets Idécorés/ d'images lvenues/ par la voie du sud au début de la transmission du boud. dhisme len Chinel.

Issu d'une coopération sino-japonaise commencée en 1991, il contient les descriptions et photographies en couleurs de 127 objets datant du II" au Iv" siècle. 120 proviennent de Chine, 7 du Japon. Il est précédé par deux préfaces, l'une de M. Liang Baiquan ${ }^{(2)}$, directeur du musée de Nankin, l'autre de M. Shigaraki Shunmaro ${ }^{(3)}$, recteur de l'Université Ryūkoku (Kyōto). Chaque preface est publiée à la fois en chinois et en japonais. Les notices des planches sont en chinois uniquement. II manque seulement une carte des lieux de découverte.

Les objets présentés dans ce catalogue sont censés être des témoins de la transmission du bouddhisme en Chine du Sud. les auteurs du catalogue pensent qu'ils sont de fabrication chinoise, mais qu'ils témoignent d'influences bouddhiques venues non pas du Nord, par la terre, mais du Sud. D'où le titre du catalogue.

Ces objets proviennent du Bas Yangri [royaume des Wu (222-280)] et du Sichuan [royaumes Shu (221-263)|. A Leshan ${ }^{(4)}$, au Sichuan, deux tombes découvertes en 1940 (Catalogue, $\mathrm{n}^{\text {os }} 1$ et 2) contiennent chacune une image de Buddha d'origine manifestement gandharienne (fig. 1) ${ }^{2}$. De la même province proviennent des objets en bronze appelés yaoqian-shu $u^{(5)}$, "arbre à secouer de l'argent", datés de l'époque Shu $\left(\mathrm{n}^{0 \mathrm{~s}} 8\right.$ $15)^{3}$. Il s'agit d'un axe vertical sur lequel sont fixés de façon symétrique, à gauche et à droite, des objets plats interprétés comme des pièces de monnaie. Parfois ces «pièces de monnaie" sont recouvertes par deux insectes, l'un à gauche, l'autre à droite, ressemblant à des cigales. Sur l'axe, entre les deux ensembles de "pièces de monnaie», se trouve parfois appliqué un personnage debout, en relief. Il est revêtu d'une grande robe. Chacune des mains tient un pan de la robe. Ces personnages rappellent aussi les Buddha du Gandhāra. I.e catalogue comprend aussi un objet en terre cuite prove-

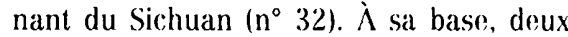
dragons se faisant face de chaque côté d'une pièce de monnaie. Au-dessus, un

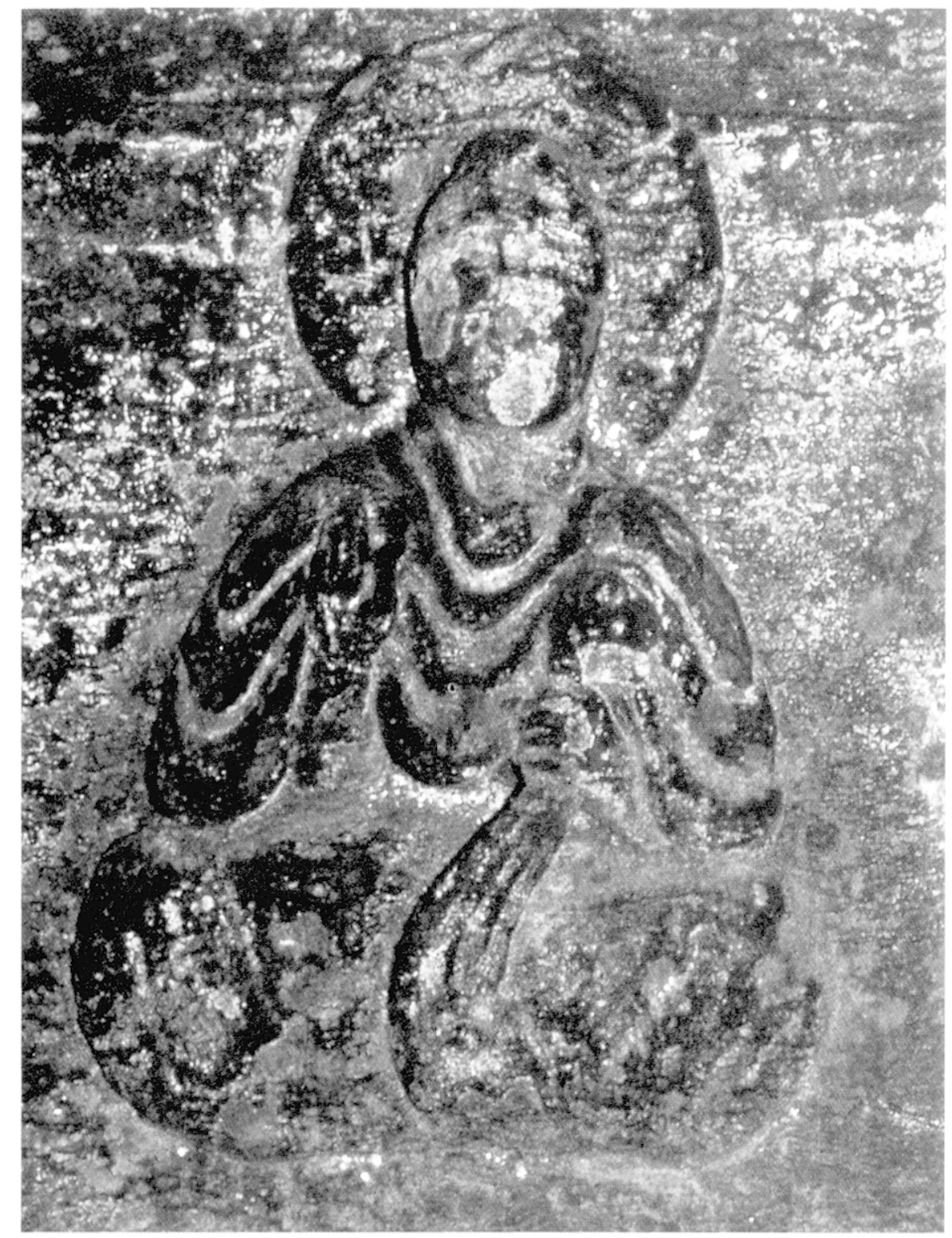

Fig. 1. Buddha en relief $(11.37 \mathrm{~cm})$ sur le linteau de la porte de la chambre du fond d'une tombe (IIr's.) de Mahao à Leshan (Sichuan). D'après le Catalogue $n^{\circ} 1$. 


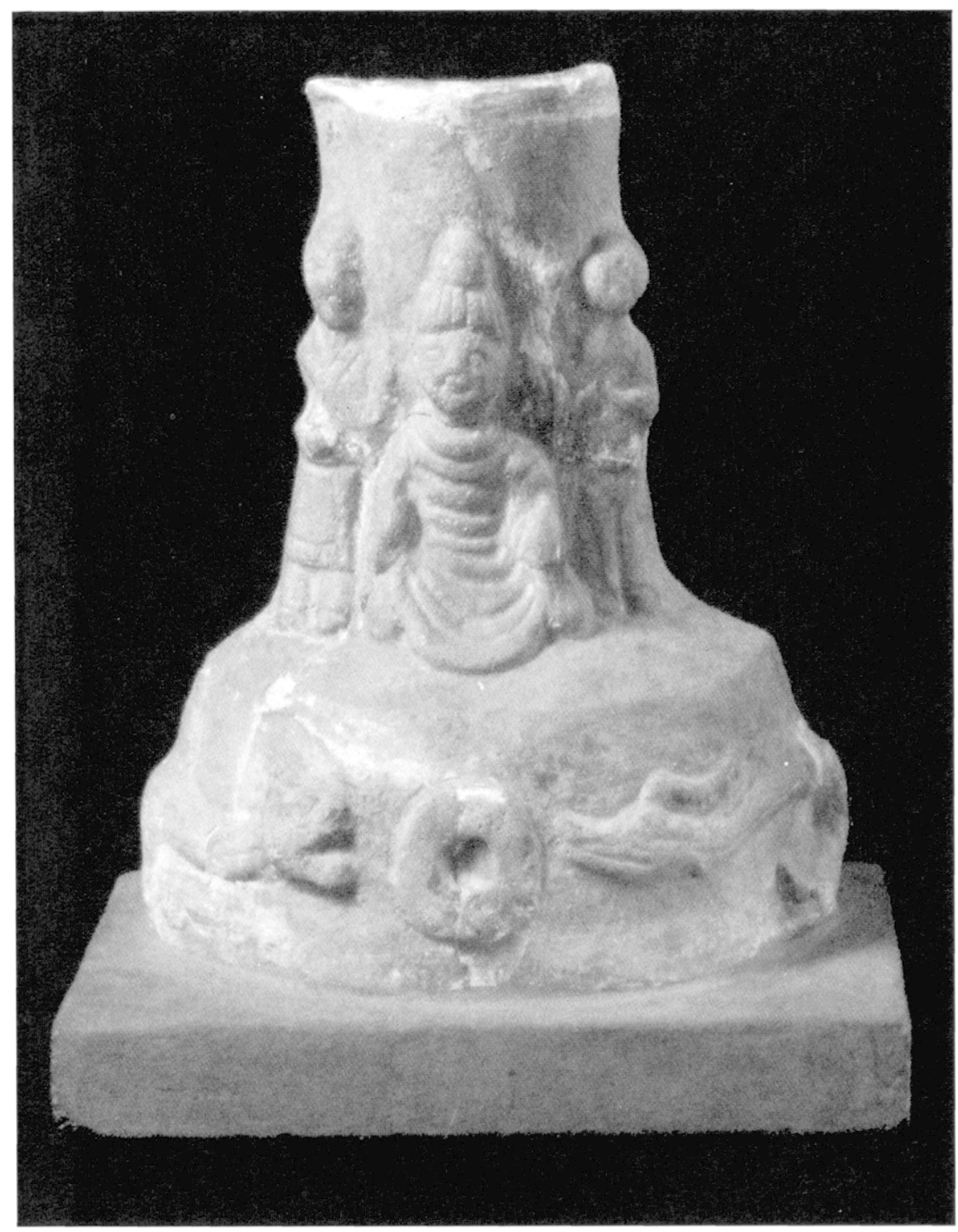

Fig. 2. Objet en terre cuite $(11.21,4 \mathrm{~cm}$, diamètre du fond : $19.3 \mathrm{~cm}$ ). découvert dans une tombe (début IIt" s.) de Pengshan (Sichuan). l)'après le Catalogue $n^{\circ} 32$.

Buddha assis entre deux personnages debout. Les personnages debout sont habillés à la chinoise. Le Buddha est d'apparence indienne. Sa main droite semble faire l'abhaya-müdra; la gauche tient un pan de sa robe (fig. 2). Du Sichuan provient aussi une représentation de stüpa entre deux fleurs datée également du III" siècle $\left(\mathrm{n}^{\circ} 3\right)$.

un pendentif en bonze doré portant une image qui pourrait être celle d'un bodhisattva sur un lotus provient du llubei (n $\left.{ }^{\circ} 16\right)$. Il est daté de l'an 262 car le contrat d'achat de terre (diquan $\left.{ }^{(7)}\right)$ trouvé dans la même tombe porte cette date ${ }^{4}$. Selon $M$. Cheng Xinren, cette image serait la plus ancienne image bouddhique sûrement datée découverte sur le sol chinois (fig. 3$)^{5}$.

On s'est longtemps demandé pourquoi les miroirs de l'époque des Trois Royaumes (220)-280) décorés d'images bouddhiques se trouvent seulement au Japon (exemples re- produits aux $n^{\text {os }} 17-19$ et $26-30$ du Calalogue) bien qu'ils y aient été importés de Chine. Selon M. Wang /hongshu, ces miroirs auraient été fabriqués au Japon par des artistes chinois venus du royaume des Wu (222-280) dans la Chine du Sud et non pas du royaume des Wei (220-265) dans la Chine du Nord". En tout cas des miroirs d'un autre type, appelés foxiang kuifeng jing ${ }^{(13)}$ « miroir orné d'images bouddhiques. d'animaux mythiques et de phénix», précédemment connus par des exemplaires de musées en Allemagne, aux États-Unis et au Japon, ont été aussi découverts ces dernières années en Chine, au Zhejiang, au Jiangsu, au Jiangxi, au Hubei et au Ilunan ${ }^{7}$. Le catalogue reproduit celui du musée national de Tókyo $\left(n^{\circ} 22\right)$, et quatre exemplaires de (hine, trois provenant d'Eahou ${ }^{(15)}$ dans le llubei $\left(\mathrm{n}^{\mathrm{os}} 21,23\right.$ et 24$)$ et un de Jingan ${ }^{116)}$ dans le Jiangxi $\left(n^{\circ} 25\right)$. Ce dernier a été découvert dans une tombe dont une brique porte la date de l'an 288 .

Les miroirs trouvés à Ezhou sont bien plus nombreux. Je crois donc utile de signaler un autre recueil de ces trouvailles, publié par Wenwu chubanshe de Pékin en 1986, édité par les musées de la Province de Hubei (Hubeisheng bowuguan) et de la ville d'Ezhou (Ezhoushi bowuguan) : licheng Han Sanguo Liuchao tongjing (17) («Les miroirs en bronze des Han, des Trois Royaumes et des Six Iynasties III av. notre ère - $N^{2} s$. de notre èrel Idécouverts à la sous-préfecturel d'licheng"). l'ouvrage contient les photographies en noir et blanc: et les estampages de 124 miroirs classés stylistiquement et chronologiquement : Han occidentaux (206 av. notre ère - 8 de notre ère : pl. 1-4), époque de Wang Meng (9-25: pl. 5-9), Ilan orientaux (25-220; pl. 15-55) et Six Iynasties (220-589 ; pl. 56-124). Les notices 144 pages] sont détaillées. lilles sont parfois accompagnées de dessins du décor de miroirs. les inscriptions sur les miroirs ont été systématiquement relevées. Des miroirs qui portent une date, six sont des Ilan orientaux, quinze sont des $\mathrm{Wu}$, de 225 à 267, deux enfin sont de la deuxième année de l'ère Iluangchu (221) des Wei. Le plus ancien est daté de 167. Les planches d'illustrations sont précédées par deux bons articles de MM. Yu Weichao ${ }^{(18)}$ et Guan Weiliang ${ }^{(19)}$. Le foxiang kuifeng jing, $\mathrm{n}^{\circ} 21$ du Catalogue se trouve dans cet ouvrage sous le numéro 81. Il est reproduit avec quelques autres miroirs analogues appelés siye bafeng jing ${ }^{(20)}$, «miroir [moulé de motifs] de quatre pétales et huit phénix» (n ${ }^{\text {os }}$ 77-82). Mais scul ce numéro 81 comporte quatre images bouddhiques, trois d'un Buddha assis on méditation sur un siège de lion et un lotus ; une montrant un bodhisattva pensif, assis en lalitāsäna devant lequel est agenouille un personnage aux mains jointes, derrière le bodhisattva, un autre personnage debout tient un parasol (fig. 4). Un autre miroir d'licheng est

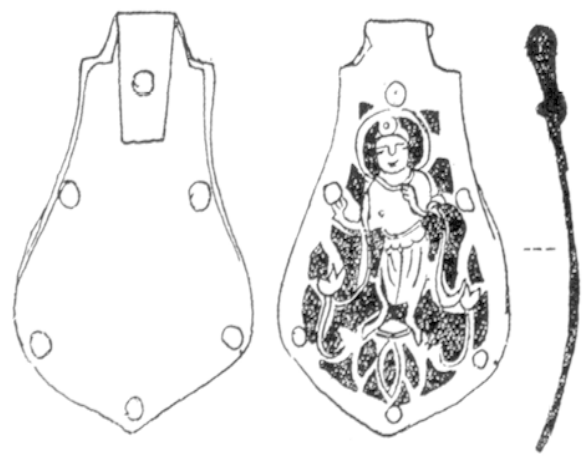

Fig. 3. Pendentif en bronze doré $(1.5 .5 .5 \mathrm{~cm}$.

l. $3.1 \mathrm{~cm}$. ep. $0.1 \mathrm{~cm})$. procenant d'une tombe de llubei datée de l'an 262. D'aprés (iheng Vinren.

Xiandai foxue $1964-2: 11$. 


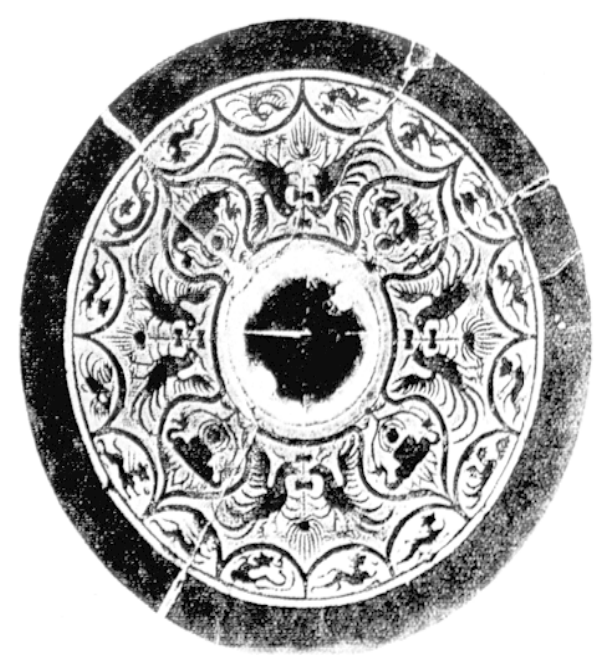

Fïg. 4. Miroir orné d'images bouddhiques

découcert à Ezhou (Hubei) (I). $16.3 \mathrm{~cm}$. ép. $0.4 \mathrm{~cm})$.

$D^{\prime}$ 'après Echeng Han Sanguo liuchao tonging. $n^{\circ} 81$ d'un tout autre style ( $\mathrm{n}^{\circ}$ 102). Il semble avoir pour décor un petit Buddha assis au milieu d'animaux et de divinités comme en portent les miroirs des Han et des Six Dynasties. L'image du Buddha est difficile à voir sur la photographie, mais $M$. Wang Zhongshu en certifie l'existence ${ }^{8}$.

Le Catalogue d'objets Idécorés/ d'images lvenues] par la voie du sud au début de la transmission du bouddhisme len Chinel regroupe de très nombreuses jarres utilisées entre le $\mathrm{III}^{\mathrm{e}}$ et la première moitié du IV ${ }^{\mathrm{e}}$ siècles comme mingqi ${ }^{(21)}$, «objets funéraires». C"est du moins la supposition généralement faite. Ce type de jarre est appelé dans le catalogue : duisuguan ${ }^{(22)}$, "jarre sur laquelle sont appliqués des éléments moulés». Les archéologues chinois donnent aussi d'autres appellations à ces jarres, telles hunping ${ }^{(23)}$, «jarre pour l'âme » ou "urne funéraire", qu'adopte Wu Hong, ou gucang ${ }^{(24)}$, "grenier». Les sa- vants japonais les appellent shinteigo ${ }^{(25)}$. "vase de pavillon divin " parce que la partie supérieure du vase évoque selon eux le monde divin où le défunt séjournera ${ }^{9}$.

l'embouchure du duisuguan, en effet. est souvent recouverte par un pavillon d'un ou plusieurs étages du style des Han. On trouve fréquemment aux quatre coins du pavillon un petit vase et des figurines moulées d'hommes, d'oiseaux et d'autres animaux. Les figuriness humaines représentent souvent des personnages de type non chinois (huren), parfois en position acrobatique ou tenant un instrument musical. Parmi ces éléments moulés, on trouve aussi l'image du Buddha (fig. 5 et 6). Celui-ci est assis sur un siège de lotus orné de deux têtes de lion, une sur chaque côté. Le Buddha est en position de méditation. Ses deux mains jointes sont presque recouvertes par la robe qui lui couvre les épaules. Le style de ce Buddha en méditation est assez voisin de celui des Buddha appliqués sur le prétendu reliquaire de Kanișka de Shāh-jī-kīdherī (près de Peshawar), daté de la fin e $^{\text {er }}$ ou du I" $^{\mathrm{e}}$ siècle ${ }^{10}$. Le même style du Buddha peut se voir aussi sur les deux Buddha appliqués dos à dos sur un axe vertical. Cet objet en bronze se trouve aujourd'hui au Musée national de Tōkyō. Il proviendrait du Pakistan et daterait des $\mathrm{v}^{\mathrm{e}}$ et $\mathrm{v}^{\mathrm{e}}$ siècles (fig. 7$)^{11}$

Les Buddha sont souvent disposés sur la partie basse du pavillon qui couvre l'embouchure du duisuguan. Ils sont groupés par deux, trois, quatre ou plus. On peut trouver un Buddha isolé aux quatre coins du toit, ou au centre de celui-ci, ou sur les étages du pavillon. Des images de Buddha analogues sont appliquées sur la panse de la jarre avec d'autres représentations (poisson, phénix, tête d'animal qui servent à décorer les poignées de porte, unicorne, etc.) (fig. 8).

Quelques jarres de ce type se trouvent dans des collections de musées aux ÉtatsUnis. M. Ho Wai-kam l'avait déjà signalé dans un article en $1961^{12}$. Mais on ignore souvent la provenance exacte de ces pièces. Une bonne douzaine des duisuguan décorés d'images du Buddha publiés dans le Catalogue provient de tombes datées de 273 à 322 par des inscriptions gravées sur les briques de celles-ci. Le duisuguan luimême est parfois daté. C'est le cas des $\mathrm{n}^{\mathrm{os}} 89,90$ et 96 du Catalogue où la date est gravée sur une stèle portée par une tortue. l'année inscrite sur le $n^{\circ} 89$ est l'an 291. Le $\mathrm{n}^{\circ} 90$ date de $292^{13}$. Sur le $\mathrm{n}^{\circ} 96$, seul est visible le nom de l'ère Yuangang (291-299). Les stèles indiquent aussi le lieu de fabrication de ces poteries : Kuaiji ${ }^{(31)}$, Shangyu ${ }^{(32)}$ et Shining ${ }^{(33)}$, tous dans le Zhejiang ${ }^{14}$. I.es voux à l'intention des descendants, obtenir un poste de haut fonctionnaire, vivre dans le bonheur et la joie sans fin, etc. sont des

Fïg. 5. Duisuguan (détail) (III. $40,5 \mathrm{~cm}, l .27,5 \mathrm{~cm}$.

découvert dans une tombe de Huzhou (Thejiang)

datée de l'an 288.

D'après le Catalogue $n^{\circ} 87$. 


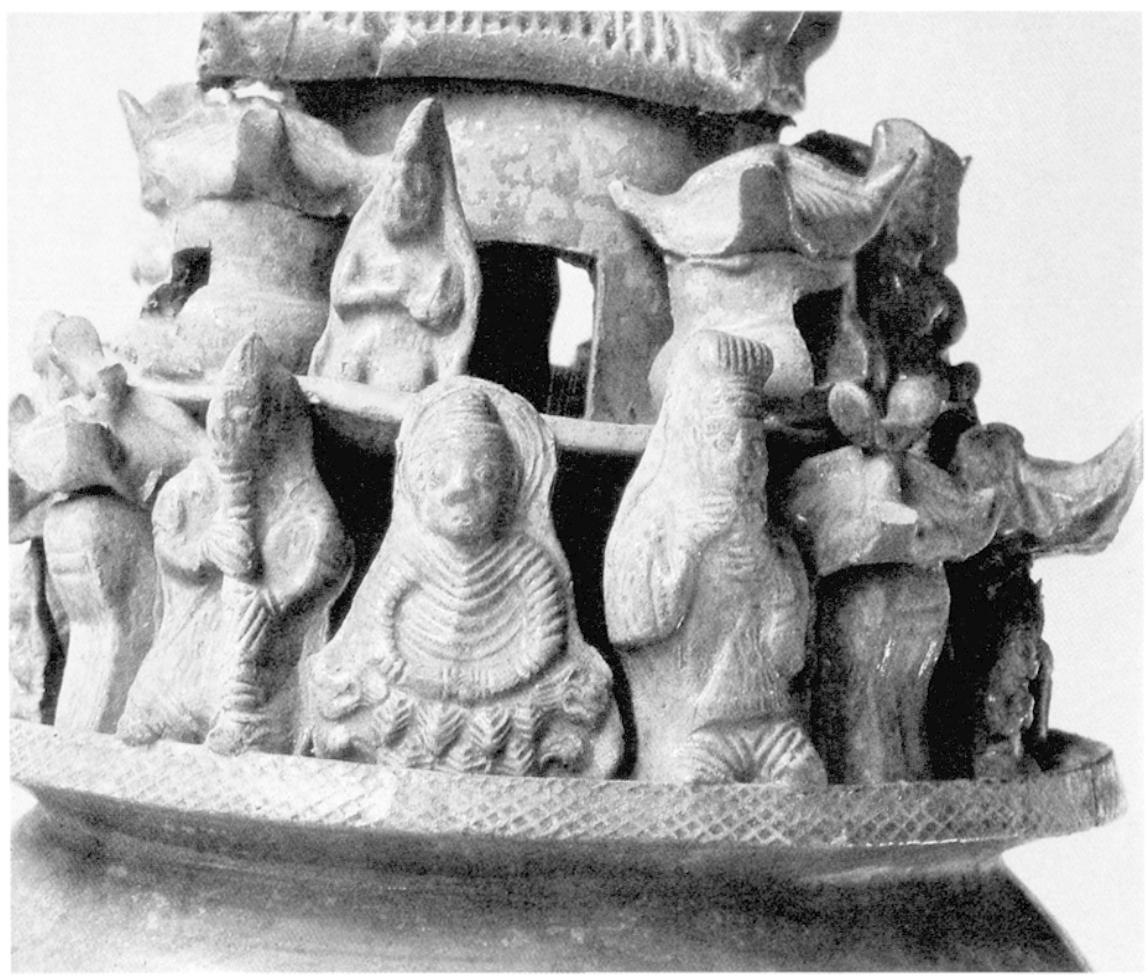

I.̈g. 6. Duisuguan (détail) $(I I .53 \mathrm{~cm})$.

découvert dans une tombe de Jinhua (Zhejiang) construite entre 266 et 316.

D'après le Catalogue: $n^{\circ} 98$. expressions souvent gravées sur la stèle, par exemple sur les $n^{0 s} 96$ (p. 183), 101 (p. 184) (fig. 9 et 10).

Les duisuguan ont été trouvés parmi d'autres objets funéraires. Leur forme et le contenu des inscriptions assurent qu'ils jouaient un rôle dans les rites funéraires. Selon M. Ho Wai-kam, ils furent utilisées exclusivement lors du zhaohunzang ${ }^{134)}$ «rite funéraire en rappelant l'âme Idu défunt]», pratiqué durant et après les troubles de l'ère Yongjia (307-312). À cette époque, des Chinois du nord se réfugièrent dans le sud du Yangzi, région où fut découverte la plus grande partie de ces duisuguan. Beaucoup perdirent la vie en chemin. la pratique de ce rite funéraire, devenue très populaire, fut interdite en 318 par la maison impériale des Jin qui se réinstalla alors dans le sud. M. Ho propose donc d'appeler ces jarres lingzuo ${ }^{(35)}$ ( $«$ the seat of spirit») ou huntang ${ }^{(36)}$ (« the hall of soul»), expressions qui se trouvent dans les décrets des Jin $^{15}$. Wu Hong refuse cette hypothese ${ }^{16}$. Il est certain en eff'et que l'utilisation des duisuguan couvre une période
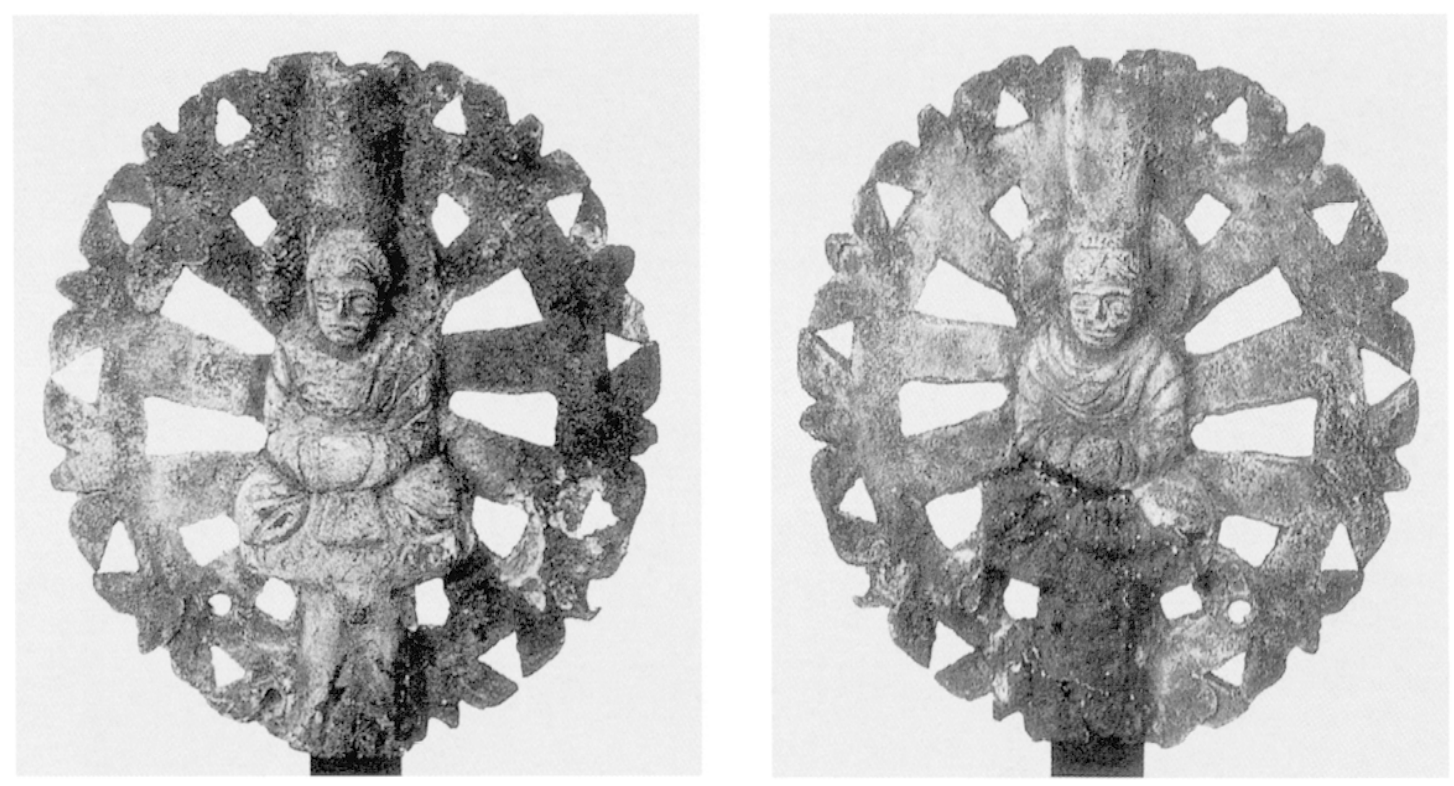

lïg. 7. Objet en bronze (II. 12,3 cm), portant deux Buddha adossés $\left({ }^{\prime \prime} " \mathrm{~s}.\right)$.

Provenance inconnue. conservé au Musée national de Tókyō.

I) aprés (hūgoku koshiki kindōbutsu to (hūó Tơnan Ajia no kindōbutsu, p. 17.

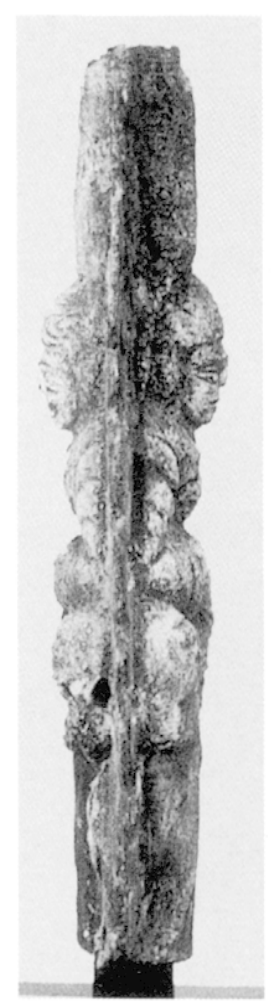




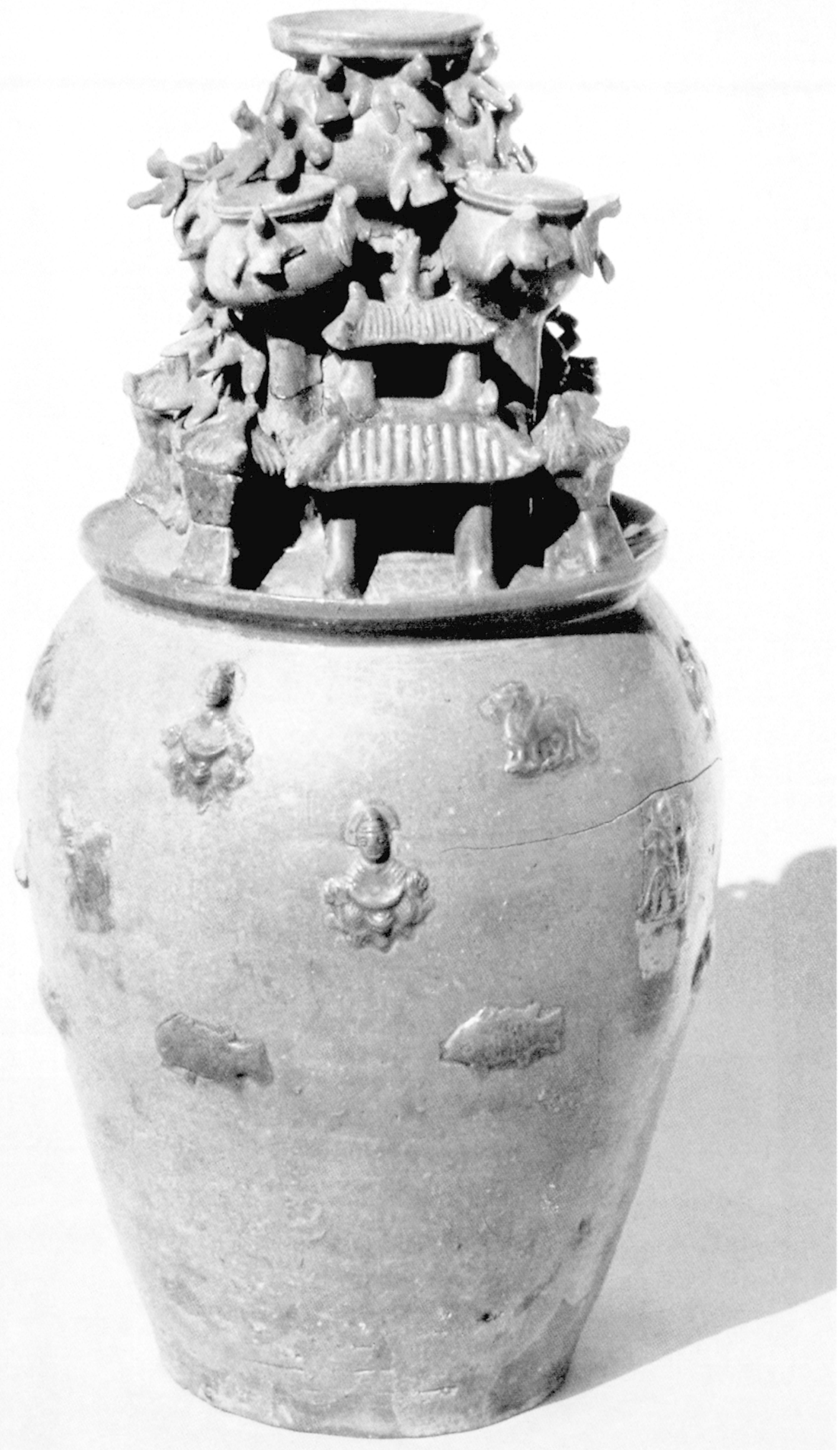

Fïg. 8. 1)uisuguan

(II. $46 \mathrm{~cm} .1 .27 \mathrm{~cm}$

découvert dans une tombe de Quzhou ('Thejiang)

construite

entre 222 ef 280

D)après le Catalogue

$n^{\circ} 61$. 


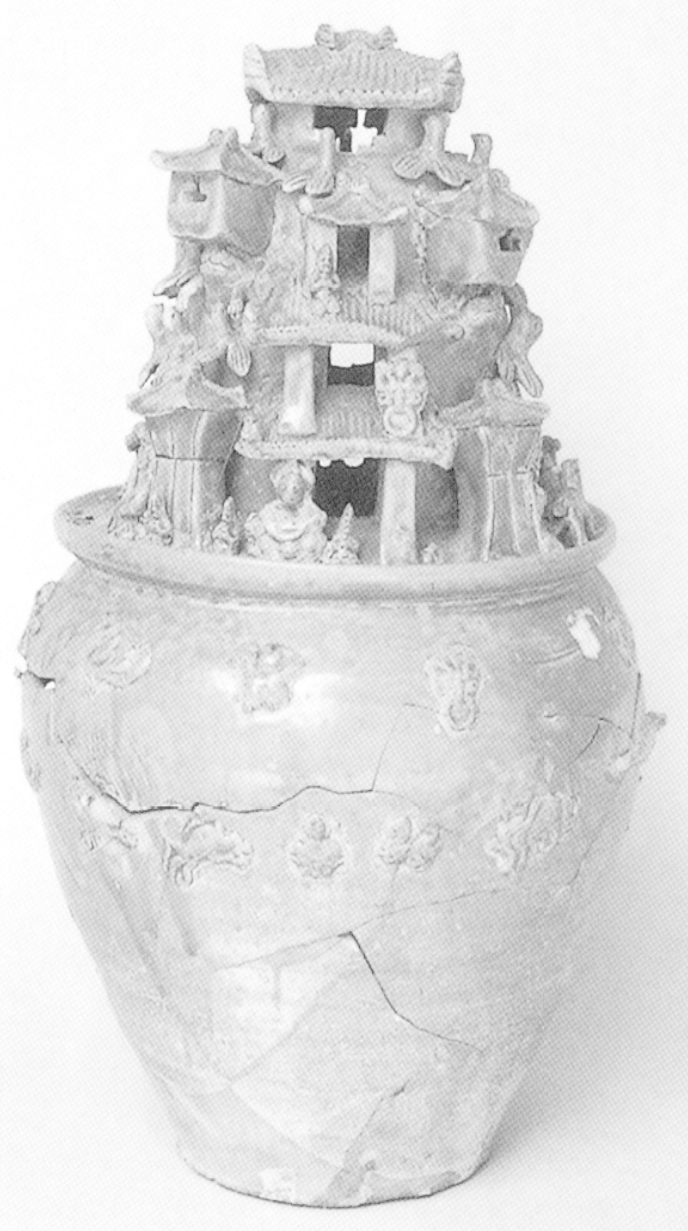

Fïg. 9. Duisuguan (II. 57,8 cm),

découvert dans une tombe de Wuxian (Jiangsu) construite entre 291 et 299.

D'apres le Catalogue $n^{\circ} 96$.

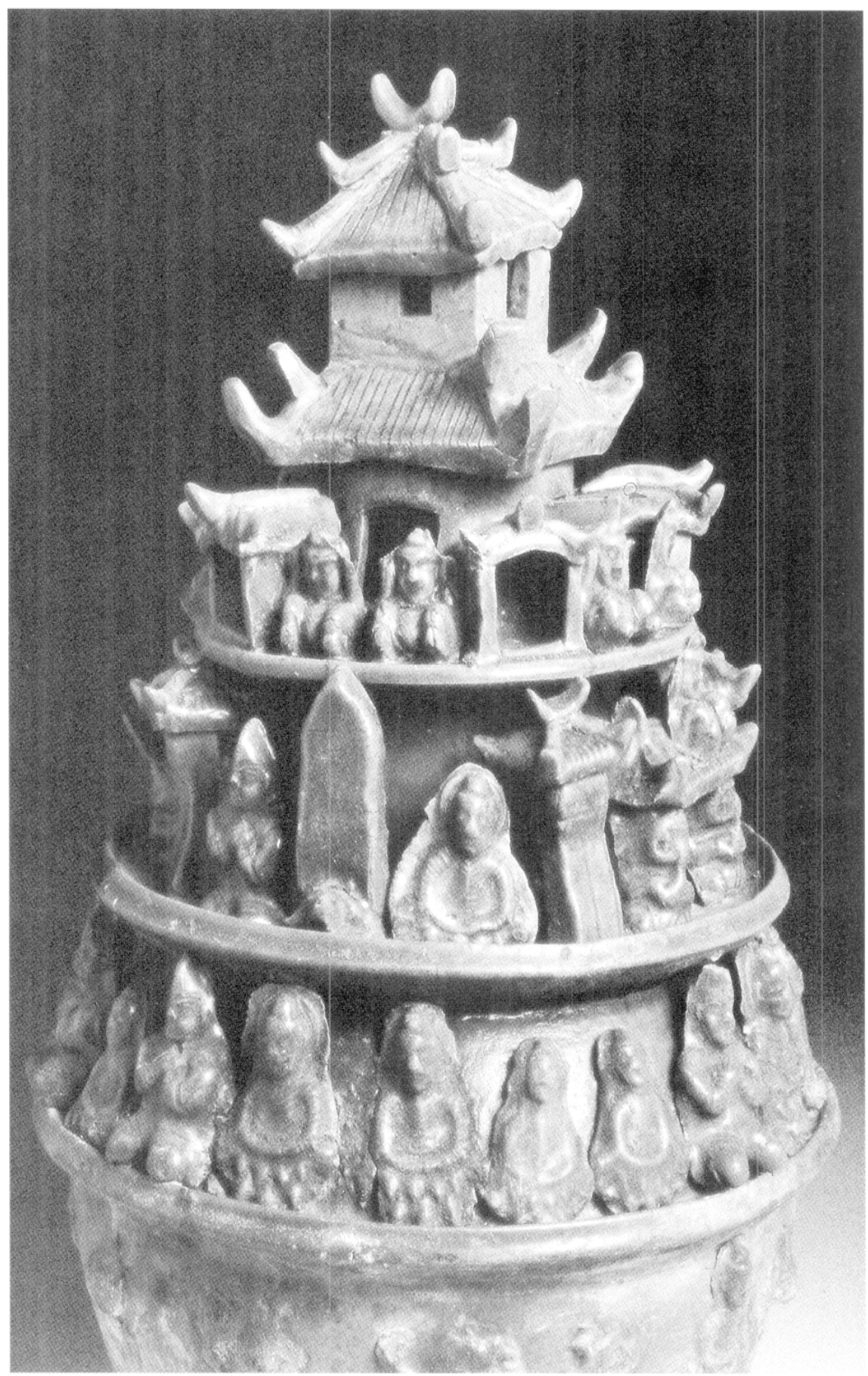

Fig. 10. Duisuguan $(11.48,6 \mathrm{~cm}$, diamètre du fond: $15 \mathrm{~cm}$ ), découvert à Wuxian (Jiangsu), daté entre 266 et 316. D'après le Catalogue $n^{\circ} 101$

réinstallation de la famille impériale des Jin dans le sud, l'arrivée de populations du nord eut des conséquences sociales et politiques dans cette région. La pratique des rites funéraires des nobles des anciens $\mathrm{Wu}$ changea, et l'objet essentiel de ce rite - le shinteigo (duisuguan) - sortit d'usage ${ }^{17}$.

Des jarres funéraires de type voisin sont connues ailleurs en Chine, au Guangdong, au Fujian, au Ilunan et au Jiangxi. Elles datent des Tang, des Song, des Yuan et des Ming ( $\mathrm{VII}^{\mathrm{e}}$-xvII ${ }^{\mathrm{e}}$ s.). Dans le Guangdong, les jarres de l'époque des Tang, selon M. Yang Hao ${ }^{(39)}$, proviennent de tombes faites pour une réinhumation. Ce type de tombe ne contient qu'une, parfois deux jarres qui renferment les ossements ou des grains de céréale $^{18}$. Ces jarres du Guangdong ont donc été utilisées comme urnes funéraires. L'une de ces urnes porte sur sa partie supérieure un pavillon central à étages. $\grave{A}$ chaque angle de la façade se trouve un stūpa à cinq étages. De chaque côté de la porte, quatre personnages auréolés, vêtus de robes chinoises, sont disposés l'un derrière l'autre. Ils tiennent chacun un instrument de musique. Ce décor peut facilement s'interpréter comme représentant une de- 


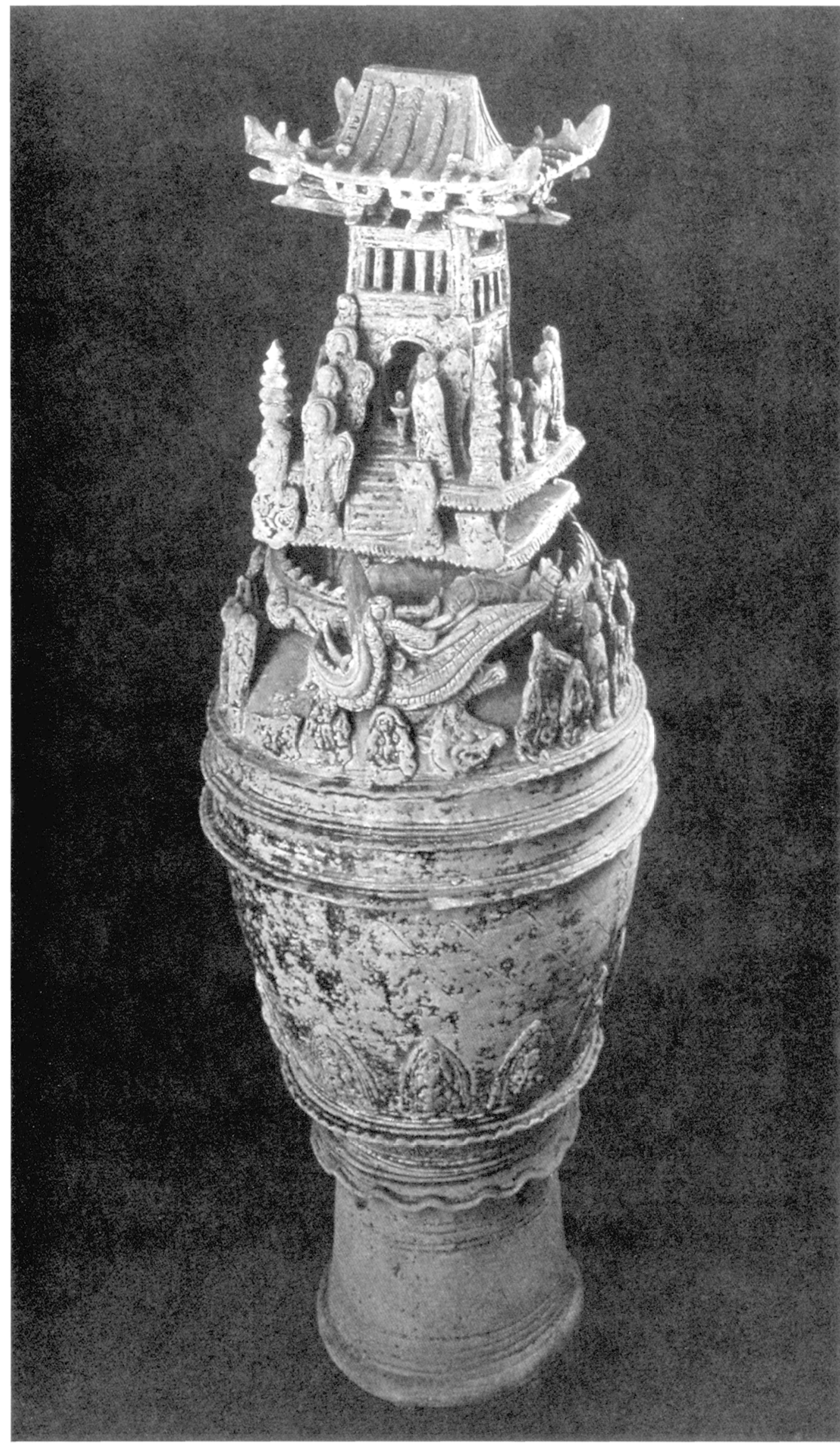

Fig. 11. Lirne funéraire de l'époque des Tang. décomuerte dans la sous-préfecture de Xinhui 
meure bouddhique paradisiaque ou une scène de cortège funéraire bouddhique (fig. 11) ${ }^{19}$. On connaît des jarres de ce type, fabriquées par paires, mais rares sont celles qui comportent des éléments bouddhiques. Leur décor relève de croyances proprement chinoises : disques marqués des caractères ri, "soleil" et yue, "lune"; animaux des quatre orients, Dragon bleu (Est) et Guerrier noir (xuanwu : Tortue et Serpent [Nord]), Tigre blanc (Ouest) et Oiseau rouge (Sud) ; divinités chinoises, parfois au nombre de douze. Iorsque la paire est conservée, on constate que le dragon, le soleil, la tortue et le serpent sont sur l'une et le tigre, la lune et l'oiseau rouge sur l'autre. On les appelle pour cette raison longhuping ${ }^{(41)}$, «jarres du dragon et du tigre» ou riyueping ${ }^{(42)}$, «jarres du soleil et de la lune». Deux bons exemples de ce type de jarre, datés de l'époque des Song, sont conservés l'un au musée Cernuschi (M.C. 9810-9811), l'autre au musée Fujiyurinkan de Kyōto ${ }^{20}$. M. Kominami, qui publie ce dernier, lui donne une troisieme appellation : guiyiping ${ }^{(43)}$, «jarre [servant] à prendre refuge Idans le Buddha|», mais ne la justifie pas $^{21}$. Un vase découvert dans la sous-préfecture de Zhijiang dans le Hubei pourrait être un exemple de ce type. Il a pour décor une scène funéraire probablement sinobouddhique, composée d'un cercueil et d'un autel d'offrande encadré par un groupe de sept musiciens et un autre groupe de cinq membres de la famille ${ }^{22}$.

M. Kominami constate que ces jarres funéraires n'ont plus été utilisées après la fin de la dynastie des Qing ${ }^{23}$. Mais il ne nous donnc aucune preuve autre que l'absence de trouvailles après cette date. (Or il semble bien que, jusqu'à une époque récente, les familles de pêcheurs de la région de Taizhou $^{(45)}$ ('/hejiang) utilisaient encore des jarres de ce type, quoique de forme beaucoup plus simple, dans le rite funéraire pour un parent disparu en mer. l'urne contenant l'âme du défunt était ensuite enterrée près de la côte ${ }^{24}$.

la forme des jarres des $\mathrm{II}^{\circ}$ et $\mathrm{N}^{\prime \prime}$ siècles dérive de celle des jarres de l'époque des Ilan appelées wulianguan ${ }^{(47)}$, «jarre à cinq [embouchures en forme de pots| fixés", comme l'affirment les archéologues chinois et japonais ${ }^{25}$. M. Kominami Koichirō a suggéré un rapport entre cette forme et la croyance aux cinq îles immortelles. Les Zhenmuwen ${ }^{(49),}$, «textes [de talisman] pour apaiser [des mauvais esprits del la tombe", découverts dans la région de Dunhuang attestent également quon se servait de la jarre comme d'un passage pour l'âme du défunt ${ }^{26}$.

le duisuguan eut certainement une fonction religieuse plus précise que les autres mingqi ou objets funéraires de la même tombe. Mais que signifient ces

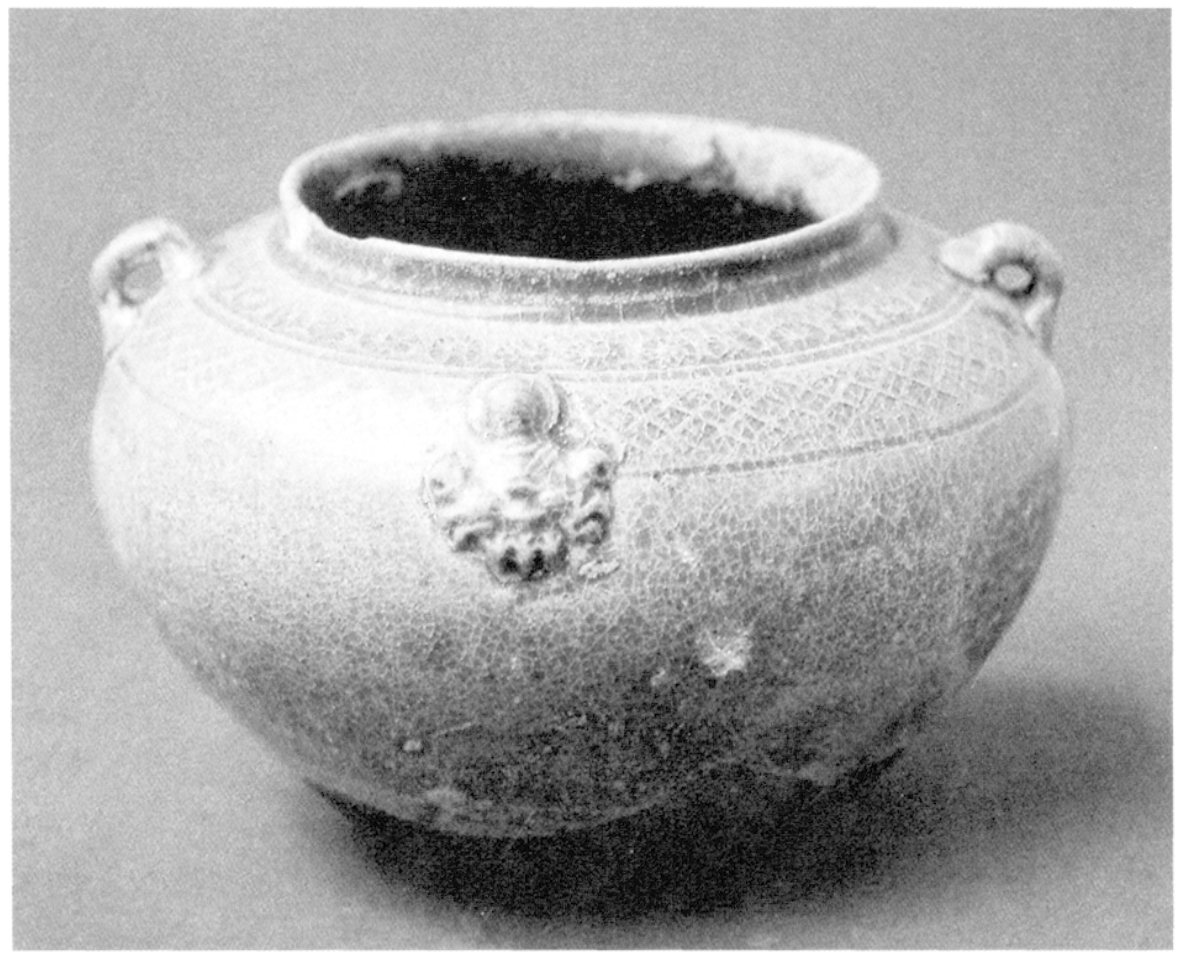

Fïg. 12. Vase en porcelaine (II. $6.5 \mathrm{~cm} .1 .11 .1 \mathrm{~cm})$, derouvert dans une tombe de Huzhou (Zhejiang) construite entre 291 et 299. D) apres le (atalogue $n^{\circ} 47$.

images du Buddha parmi les animaux fabuleux et figurines étranges? Ces scènes permettent-elles une interprétation bouddhique? Ou l'image du Buddha était-elle utilisée simplement comme décor? Sur d'autres vases de style plus simple provenant de la même région du Bas Yangæi (C'atalogue, $\mathrm{n}^{\text {os }} 38$-51), l'image du Buddha mouléc est appliquée sur la panse. Elle semble n’avoir été qu'un simple décor (fig. 12).

Depuis la deuxième moitié du premier siècle un bouddhisme mêlé de pratiques taoïstes fut pratiqué dans le nord du Bas Yangzi. Au milieu du mi $^{\prime}$ siècle, des textes bouddhiques mentionnent la construction du premier monastère bouddhique de Jiankang (actuel Nankin) au profit du moine Kang Senghui ${ }^{(52)}$, venant du Jiaozhi (nordouest du Viêtnam). A cette époque, la communauté bouddhique de la capitale des $W u$, Jiankang, pratiquait aussi la cérémonie du bain de la statue du Buddha ${ }^{27}$. Des sources bouddhiques phus tardives ( $v^{\mathrm{C}}$ et début du $\mathrm{vl}^{\mathrm{e}}$ s.), attestent la pratique du culte de l'image du Buddha. Mais le décor des duisuguan est si hétéroclite qu'il est impossible d'affirmer que les propriétaires de ces vases funéraires étaient bouddhistes. Dans les tombes qui contenaient les duisuguan portant des images du Buddha, on a parfois trouvé des miroirs en bronze, mais apparemment sans décor bouddhique ${ }^{28}$

Suite à la découverte de ces objets comportant des images bouddhiques, quelques publications chinoises récentes affirment que la première transmission du boud- dhisme se serait faite par le sud, par la mer, avant celle venue d' $\Lambda$ sie centrale ${ }^{29}$. Cette affirmation paraît exagérée. Certes, il est extrêmement important de trouver des images de Buddha datées de haute époque en Chine du Sud, mais rien ne prouve qu'elles aient été vénérées en contexte bouddhique. Comme l'affirme Shigaraki Shunmaro dans la próface du Catalogue (p. 30), ces images ou éléments bouddhiques sont isolés et indépendants; ils ne se trouvent pas dans un contexte bouddhique. De plus, on peut difficilement nier que la transmission de textes bouddhiques dans la Chine du Nord par la route du nord eut lieu avant l'apparition de ces duisuguan (III" s.). I)'autres découvertes permettront d'y voir plus clair. L'hebdomadaire publié à Pékin. Zhongguo wenwubas ${ }^{(59)}$ (Journal sur le patrimoine culturel de (hine), a récemment signalé d'autres trouvailles de ce type de jarres du III $^{\circ}$ siècle dans le Bas Yangzi ainsi que celle d'une paire de jarres du ${ }^{x} I^{e}$ siecle dans le Fujian ${ }^{30}$. Selon un article récent du même hebdomadaire, les bouddhistes du Yunnan, de l'époque de l'ancien royaume des Nanzhao ${ }^{(6)}$ (VIII"- début du $x^{\mathrm{P}}$ s.) jusqu'au début du $\mathrm{xix}^{\mathrm{e}}$ siècle, utilisaient soit une jarre soit deux ou trois jarres emboittées, pour conserver les cendres des défunts et quelques objets funéraires ${ }^{31}$.

Kt:o LIYTivi

Membre scientifique de l'FFEO 22 avenue du Président-Wilson, 75016 Paris, liranco 
Notes

1 Wi llong, "Buddhist Elements in Larly chinese Art (2nd and 3rd Centuries A.D.)". Artibus Asiae XIVII. $3 / 4$ (1986) : 26.3-352.

2 Le $n^{\circ} 1$ a été étudié par Richard limwans, "The Cave Reliefs at Ma Hao I. II". Artibus Asiae XVII. $1(1954)$ : 5-28 et XVII, $2(1954)$ : 103-129. Repris dans Wi Hong, op. cit., p. $266-267$.

3 Les n ${ }^{\text {"m }} 8.12$ sont conservés à l'Institut d'archéologie du Sichuan. I.es $n^{135} 13-15$ se trouvent at Japon : le $n^{\circ} 13$ appartient au (iroupe des biens culturels Kuboso de la préfecture dosaka an Japon ; les $n^{\prime \prime s} 14$ et 15 sont en possession des par ticuliers. Sur la signification de ces objets, voir $Y_{1}$ Haoliang, "Qianshu" "gianshuzuo" he yulong manyan zhi xi »" ("l'arbre à l"argent", "le sieg de l'arbre à l'argent» et le jeu du poisson et du dragon), Wenu'u, 1961-11:43-45.

4 llubeisheng wenwu guanli weiyuanhui, «Wu chang Lianxisi Dongwu mu qingli jianbao " ${ }^{\text {(S }}$ (Rapport archéologique sur une tombe de l'époque des Wu de l'list située au temple lianx de Wuchang Iprovince de Hubeill, Kaogu, 1959-4 : 189-190 et pl. VII-8.

5 Cim:xi Xinren, «Woguo xiancun gudai fojiao zuizao de yizun zaoxiang ${ }^{\prime(9)}$ (l.a plus ancienne image bouddhique actuellement conservée en (hine) Xiandai foxue. 1964-2: 11-12.

6 Wuvi Zhongshu. "(juanyu Riben sanjiaovuan foshoujing - da Vishida Morio xiansheng ${ }^{1101}(\dot{\lambda}$ propos des miroirs decores d'images bouddhiques et d'animaux [trouvés] au Japon - Réponses données à M. Nishlid) Moriol, Kaogu, 1982-6: 6.30 639, voir aussi $i d$, "Guanyu Riben sanjiaoyua

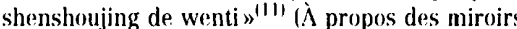
décores d'images de divinités et d'animaux Itrou vés| au Japon), Kaogu, 1981-4: 346-358. les ar ticles de Wiv; Zhongshu sur les miroirs publies dans le Kaogu et le Iluaxia kaogu ont été rassemblés et traduits en japonais : Sankakubuch shinjükyo -121 $^{-1}$ (Les miroirs ornés d'images de divinités et d'animaux aver le motif de triangle en bordure). Tōkyō, 1992.

7 Wive thongshu "Lun Wu Jin shiqi de foxian kuifengjing - wei jinian Xia .Vai xiansheng kaogu wushinian erzuo ${ }^{11+1}(\dot{A}$ propos des miroirs déco res d'images bouddhiques, de fleurs et de phénix de l'epoque des Wu et des Jin III"-11" s. I - à l'oceasion des cinquante années d'archéologie de M. XI Nai), Kaogu, 1985-7:636-643 + pl. 4-7. (es decors de miroirs (animaux divinités chinoises et images bouddhiques) sont discutés dans W1: Ilong. op. cit., p. 275-283.

8 Wiv; Thongshu, op. cit. Kaogu, 1982-6: 634. Repris dans Wi Hong, op. cit., fig. 35

9 Wi llong mentionne l'appellation japonaise en reprochant aux Japonais de ne pas donner de justification (op. cit., p. 286). (Quelques articles en japonais ont été publiés depuis : Hosicillm Michitaka, "(jo Shin (Seishin) bo shutsudo no shinteigo - Keifu oyobi ruikei o chūshin $\mathrm{ni}-n^{(26)}$ (Shinteigo découverts dans les tombes des Wu et des Jin occidentaux - étude sur l'évolution de leur formel. Kökogaku zasshi, 71-3 (1986) : 61-77 ; Koмmsu Ichirō, "Kokei no uchū" ${ }^{|2 i|}$ (The Cosmos within a Jar), Töhö qakuhō, Kyōto, vol. 61 (1989) : 16.5-221 et idem, "Shinteigo to Tögo no bunka" ${ }^{(2 \mathrm{~s})}$ (Aspect of Eastern Wu Culture as Reflected in the "Ilunping" Spirit Bottles). Töho gakuho, Kyoto, vol. 6.5 (1993) : 22.3-311.

10 Lobjet. découvert au début du siecle. a eté tries souvent reproduit et discuté. Pour l'inscritpion et la date, voir B.N. MI kill:RIl:. "Shāh-ji-kī-dheri Casket Inscription". The British Museum Quarterly XXVIII-1-2 (Summer 1964) : 39-46 + pl. XIVXVIl et Gerard li sivily, «Numismatic and Epigraphic Evidence for the Chronology of Early Gandharan Art", Investigating Indian Art, Berlin, 1987. p. 67-88.

11 L'objet est haut de $12,3 \mathrm{~cm}$. Chügoku koshiki kindōbutsu to (hüō Tönan Ajia no kindōbutsu $\iota^{20 y}$ Les Buddha en bronze de forme ancienne de la chine et les Buddha en bronze de l'Asir Centrale et de l'Asie du Sud-list), éd. par Izumishi Kuboso kinen bijutsukan (musée des Beaux Arts Kuboso de la ville d'lzumi). 1988 , p. 17

12 Ho Waj-kam, "llun-p'ing : the Lrn of the Soul". The Bulletin of the Cleveland Museum of $\mathrm{Art}$. vol. $48, n^{\circ} 2$ (1961) : 26-34. On en trouve aussi un exemple dans la collection de (harlotte (. et Jishn C. Weber au Metropolitan Museum of Art, $c f$ Mj-

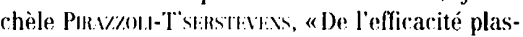
tique à la productivité: les grès porcelaineux du

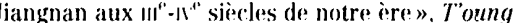
Pao, t. I.XXXIV, fase. 1-3 (1998) : 50, fig. 8.

13 D'après 'Zlmo 'Zhixin la date inscrite, "au mois intercalaire de la deuxième année de lère Yuangang" (292), est une erreur pour la troisième année (293) rar il n'existe pas le mois intercalaire pour l'an 292: «Jiangsu Wuxian Shizishan Xijin mu qingli jianbao $)^{(? 0)}$ (Rapport archéologique sur les tombes des Jin occidentaux au Mont de Lion dans la sous-préfecture de Wu de la province du Jiangsu). Wenu'u ziliao congkan. 1980-3: 134135.

14 Kuaiji correspond à l'actuelle Shaoxing. Shining se trouve dans le sud-est de l'artuelle sous-préfecure de Shangyu of Zllwivi Zhixin loc cit p. 136

15 Ho Wai-kam. op. cit. p. 31-33 et Ouan Jin wen ${ }^{133}$ (Recueil complet des Jin), juan 107, 124, 132 ot 140. éd. par Y.w Kejun. Quan Shanggu Sandai Qin Ilan Sanquo liuchao u'en ${ }^{13 \times)}$ (Recuril complet des Trois loynasties de la haute antiquité. des (Qin, de Han des Trois Royaumes at des Six Dynastiesl. Beijing, 1958.

6 Wi llong, op. cit. p. 287-288

17 Kosmvivi Ichirō, Tōhö gakuhō, vol. 65 (1993) : 303.

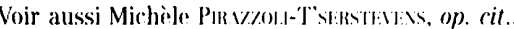
p. $56-57$.

18 Cuangdong chutu Jin zhi Tang wenu $u^{(4) 1} \mid \mathrm{Ar}$ chaeological linds from the Jin to the Tang Periods in (juangdong). The Chinese Lniversity of Hong Kong. 1985, p. 27.

19 Guangdong chutu Jin zhi Tang Ue'nu'u, pl. 82 (photo en couleur), p. 206 (texte et dessin) et p. 207 (photo en noir et blanc). Voir aussi p. 208. 211 deux urnes funéraires de style plus simple.

20 Arts Asiatiques XIIV (1989), "Activités du musée Cernuschi ", p. 120 et lig. 5 (p. 121): (hine connue et inconnue. Dix amnées d'acquisitions an Musé Cernuschi 1982-1992. musee Cernuschi. Paris 1992, p. 148-150 (149 : photos) dont la noties signale egalement d'autres vases de ce type dévou verts en Chine. Pour la paire de lujiyurinkan, voir KovIxay Ichirō, Tohö gakuho, vol. 61 (1989) : 18.3 (photos) et 185.

21 Kovmsavil Ichirō. Töhö gakuho, vol. 61 (1989) : 185

22 Zhijiangxian wenhuaguan, "llubej \%hijiangxian faxian Songchao shigi taosuguan ${ }^{(+1+1}$ (Lin suguan en terre cuite de lépoque des Song découvert dans la sous-prefecture de \%hijiang du Hubei). Kaogu, 1989-8: $763-764$.

23 KonwavI lehirō, Töhö gakuho. vol. 61 (1989) 184-185 et 209

24 Ce rensejgnement ma été communique par M. I. Mingyou ${ }^{(+6)}$, professeur à l'Eniversité de \%hejiang. témoin de cette pratique dans son enfance.

25 Voir par ex. Zhenjiang bowuguan. "Zhenjiang Dongwu Xijin mu " ${ }^{\mid+\times)}$(L.es tombes des Wu orienlaux et des Jin occidentaux à thenjiang). Kaogu, 1984-6 : 544 : Hlsili,W: Michitaka, op. cit.. p. 73 75. Kondsum Ichiro le montre clairement par des dessins de jarres datées, Tōho gakuhó. vol. 65 (1993) : 271-272. Voir aussi Michele PIn.vint.Il"siRstiviss, op. cit., p. 46 (dessins) et 48.

26, Dunhuang wenwu yanjiusuo kaoguza, "Dunhuang Jin mu " "To" (Tombes des Jin), Kaogu, 1974 3:196 (fig. 13) et 198; Henansheng bowuguan. "Linghao Zhangwan Ilan mu" "\$1" Tombes des Han à Zhangwan [de la sous-préfecture] de lingbao Idans le Henanll. Wenu'u, 1975-11:79-80 KowivinI Ichirō, Töhō gakuhō, vol. 61 (1989): $167-176$

27 Tavi Yongtong, IIan Wei Jiangjin Yambeichan fojiaoshi ${ }^{t 33}$ (Hlistoire du bouddhisme des llan, des Wei, des I)eux Jin et des Six Dynasties), Shanghai. 1938 , p. 39 et 99 et Eric: 7owilln. The Buddhist Conquest of China: The Spread and Adaptation of Buddhism in Early Medieval China, Leiden. 1959. p. $26-28$ et $51-52$

28 Dans une tombe des Jin occidentaux de Nanjing. un miroir trouve aver une jarre portant des images du Buddha a une forme qu'on considere dérivée du foxiang kuifeng jing "miroir orné d'images bouddhiques, d'animaux mythiques et de phénix" (supra), mais sans representation bouddhique (Catalogue, $n^{\circ} 981$ : Jinhua diqu wenguanhui, "Zhejiang Jinhua gufang Liuchao mu" ${ }^{1541}$ (l.es tombes anciennes des Six l)ynasties a Jinhua du \%hejiang). Kaogu. 1984-9 : 82()-822, pl. VI - 2, 5-6.

29) Wi Zhuo, "(ong kaogu yicun kan fojiao chunru Xiyu de shijian ${ }^{1.55}$ (la date de lat transmission du bouddhisme en Asie centrale d'apries les vestiges archéologiques), Dunhuangxue jikan, 8 [1985-2] 62-72 : id., Fojiao dongchuan yu Zhongguo fojiao yishu ${ }^{(56)}$ (La transmission du bouddhisme a l'est et lart bouddhique en Chinel. Zhejiang renmin chubanshe. 1991; Wi Tingqiu et \%ux; Pengnian, "lojiao haishang chuanru Zhongguo zhi yanjiu ${ }^{10 \pi /}$ (Étude sur la transmission par la mer du bouddhisme en (hine), lishi yanjiu. 1995-2: 20-

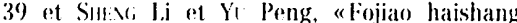
chuanru shuping $»^{15 \times 1}$ (Commentary on the Introduction of Buddhism into (hina), Haijiaoshi yamjiu (Maritime History Studies). 1997-1:61-67.

30 thongguo u'enueubao. $\mathrm{I}^{\circ} 22 \mathrm{fn}^{\circ} 587$ au totall. $22 / 03 / 1998$ et $n^{\circ} 38\left(n^{\circ} 603\right.$ au total), 17/05/1998.

31 thongquo wenuubao, $\mathrm{n}^{\circ} 49 \mathrm{(n}^{\circ} 614$ au total). $24 / 06 / 1998$. 
（1）佛教初傳南方之路文 物回錄

(2) 梁白泉

(3) 信樂峻 磨

(4) 樂山

(5) 播錢樹

(6) 于豪亮，“錢樹”“錢樹座” 和魚㛺漫衍之戲》

(7) 地 券

（8）湖北省文物管理委員 會，《武昌蓮湑寺東哭墓 清理筬報》

(9)程欣人, 《我國現存古 代佛教最早的一尊造 像”

(10)王仲殊, 《閶于日本三 角緣佛漹鏡一答西田守 夫先生》

(11)《關于日本三角緣神獸 鏡的問題》

(12) 三角緣神 獸鏡

(13) 佛像变風鏡

(14) 《論叁晉時期的佛像繁 風鏡一為記念夏震先 生考古五十年而作》

(15) 㔀 州

(16) 安

(17) 鄂城漢三國六朝銅鏡

(18) 俞偉超

（19）管維 良

(20)四葉八瓦鏡

(21) 明 器

(22) 堆 㙰

(23) 砶 瓶

(24) 墪 倉

(25) 神亭斝

(26) 長谷川道隆, 《题晉 (西

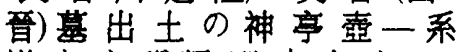
譜および類型を中心 に一»

（27）小南一郎, 《䃘型の宇 宙》

(28) 《神亭辜と東哭の文 化”

（29）中國古式金銅佛と中央 東南アジアの金銅佛

(30) 張志新, “江蘇悬縣㕰子 山西晉基清理簡報》

(31) 曾稭

(32) 上虞

(33) 始蜜
(34) 招魂葬

(35) 露

(36) 磈堂

(37) 全 晉 文

（38）覇可均, 全上古三代秦 漢三國六朝文

(39) 㛫豪

(40) 廣東出上䙳至唐文物

(41) 龍虎㼛

(42) 日月瓶

(43) 饭 依瓶

(44) 枝江㲘文化館, 《湖北 枝江縣矩現宋朝時期 陶塑 罐》

(45) 台州

(46) 李 明友

(47) 五 聯 蠸

(48)鎮江博物館, 《鎮江東吴 西晉慕》

(49) 鎮墓文

(50) 敦煌文物研究所考古 組，《敦煌晉蓦》

（51）河南省博物館，《害害張

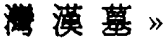

(52) 康僧會

（53）湯用䑣，漢魏兩㸸南北 朝 佛教史

(54) 金華地區文管會, 《浙江 金華古方六朝墓》

(55) 呂焯, “從考古遗存看佛 教傮入西域的時間》

(56) 佛教東傳與中國佛教 荎 術

(57) 吳廷璆, 暗彭年, 《佛教 海上: 専入中國之研究”

(58) 盛利, 于澎, “佛教海上 傳入速評”

(59) 中國文物報

(60) 南詔 